\title{
Comparative secretome analysis of cholangiocarcinoma cell line in three-dimensional culture
}

\author{
PHANTHAKARN TIT-OON ${ }^{1}$, DARANEE CHOKCHAICHAMNANKIT ${ }^{2}$, AMNART KHONGMANEE ${ }^{1}$, \\ PHANNEE SAWANGAREETRAKUL ${ }^{2}$, JISNUSON SVASTI ${ }^{1,2}$ and CHANTRAGAN SRISOMSAP ${ }^{2}$ \\ ${ }^{1}$ Applied Biological Sciences Program, Chulabhorn Graduate Institute, ${ }^{2}$ Laboratory of Biochemistry, \\ Chulabhorn Research Institute, Bangkok 10210, Thailand
}

Received July 1, 2014; Accepted August 13, 2014

DOI: $10.3892 /$ ijo.2014.2636

\begin{abstract}
Cholangiocarcinoma (CCA) is a lethal malignancy which occurs with relatively high incidence in Thailand. This cancer is often difficult to diagnose and associated with high mortality. The secretome, containing the secreted proteins from cells, are potentially useful as biomarkers of cancers. Since three-dimensional (3D) cell culture may mimic growth characteristics and microenvironment of solid tumors in vivo better than monolayer culture, we have developed culture of CCA in natural collagen-based scaffold, to enable analysis of the secretome by $2 \mathrm{DE}$. Our results indicated that CCA growth in 3D environment alters cell shape significantly and enhances extracellular matrix deposition. Interestingly, more secreted proteins were detected from 3D culture compared to monolayer culture. Secretome analysis using 2DE coupled with LC-MS/MS demonstrated 10 secreted proteins uniquely found in 3D culture. Moreover, 25 proteins were enriched in $3 \mathrm{D}$ culture compared to monolayer culture, including 14-3-3 o, triosephosphate isomerase, phosphoglycerate mutase $1, \alpha$-enolase, and L-plastin. Immunoblotting was used to confirm the presence of L-plastin in conditioned media of CCA and of hepatocellular carcinoma (HCC) cell lines. The results revealed that L-plastin, an actin bundling protein, was uniquely expressed only in the CCA cell line and could be a promising biomarker for differential diagnosis of CCA compared to $\mathrm{HCC}$.
\end{abstract}

Correspondence to: Dr Chantragan Srisomsap, Laboratory of Biochemistry, Chulabhorn Research Institute, 54 Kamphaeng Phet 6 , Talat Bang Khen, Laksi, Bangkok 10210, Thailand

E-mail: chantragan@cri.or.th

Abbreviations: CCA, cholangiocarcinoma; CDM-HD, chemically defined medium for high density cell culture; ECM, extracellular matrix; ENO1, $\alpha$-enolase; HuCCA-1, human cholangiocarcinoma cells; LCP1, L-plastin; PGAM1, phosphoglycerate mutase 1; SFN, 14-3-3 б; TPI, triosephosphate isomerase

Key words: three-dimensional culture, cholangiocarcinoma, secretome, proteomics, cancer

\section{Introduction}

Cholangiocarcinoma (CCA) is a malignant neoplasm of biliary tract epithelium, which shows progressively increased incidence and mortality over the past decades (1). Northeast Thailand has the highest incidence of CCA in the world (2), where it is most often associated with Opisthorchis viverrini infection (3). This tumor is oftentimes fatal due to late stage diagnosis as well as limited options and success with treatment. The most widely used serum biomarker for CCA is carbohydrate antigen (CA) 19-9 and carcinoembryonic antigen (CEA). However, CA19-9 is also increased in non-malignant obstructive jaundice, severe hepatic injury, and several cancers such as pancreatic and gastric cancer. In addition, the sensitivity and specificity of CEA in serum is relatively low. Novel potential serum and bile markers associated with CCA include matrix metalloproteinase-7 (4), interleukin-6 (5), and microRNA (miR-9) (6). Unfortunately, none of these biomarkers have yet to be validated in large clinical studies. As diagnosis of CCA remains problematic, developing a more effective biomarkers and therapeutic modalities could significantly increase the chance of early detection for these patients.

Proteins secreted by cancer cells are ideal specimens to investigate biomarkers since they have important roles in cell signaling and pathological developments such as differentiation, invasion and metastasis. Detection of these secreted biomarkers is also non-invasive as they are detected in bodily fluids such as blood and urine making secretomic study beneficial for not only identifying biomarkers (7-9) but also improve routine medical evaluations. However, secretome analysis of monolayer cell culture has several limitations such as low concentration of secreted proteins and contamination with cellular proteins due to cell lysis. To improve success in biomarker discovery, a more reliable and representative cell culture model may help bridge the gap between in vitro research and clinical studies (10).

Three-dimensional (3D) culture is being more extensively used since it provides a more realistic microenvironment in terms of natural physiology than conventional monolayer culture (11). Several techniques have been introduced to create in vitro $3 \mathrm{D}$ cancer models such as scaffold-based, spheroid aggregation, liquid overlay, and rotary cell culture systems. Scaffold-based 3D culture offers advantages in providing a 
structural support for cellular attachment and may modify the signaling responses of cells (12). 3D culture systems have been used in many applications including cancer biomarker discovery studies (13). Thus, the present study aimed to develop scaffold-based 3D culture of human intrahepatic CCA and to use this model as a tool to study the expression of secreted proteins by proteomic analysis. Comparative secretome analysis of 3D culture to monolayer culture enabled us to discover potential biomarkers for CCA.

\section{Materials and methods}

Cell cultures. Human cholangiocarcinoma cells (HuCCA-1), derived from a Thai patient, were grown in Ham's F-12 media (Gibco ${ }^{\circledR}$, Invitrogen, USA) (14). Hepatocellular carcinoma cells HCC-S102 established from a Thai patient were grown in RPMI-1640 (Gibco) (15) while HepG2 and SK-HEP-1 cell lines were purchased from American Type Culture Collection (ATCC) and grown in DMEM (Gibco). All cell culture media contained $10 \%$ fetal bovine serum (FBS, Hyclone Laboratories, USA), $100 \mathrm{U} / \mathrm{ml}$ penicillin, $100 \mathrm{mg} / \mathrm{ml}$ streptomycin, and $125 \mathrm{ng} / \mathrm{ml}$ amphotericin B (Gibco) and were maintained at $37^{\circ} \mathrm{C}$ in a humidified atmosphere, $95 \%$ air, $5 \% \mathrm{CO}_{2}$. In serum-free culture, FBS gradually decreased and was simultaneously replaced with chemically defined medium for high density cell culture serum replacement (CDM-HD; FiberCells ${ }^{\circledR}$ System, USA) (16).

Development of 3D culture. Scaffold-based 3D culture was established using natural collagen type I fibrils named Lyostypt $^{\circledR}$ (Braun, Germany) (17). Briefly, cells were seeded onto sterilized collagen scaffold, followed by incubation for $2 \mathrm{~h}$ to allow cell attachment. Media was gently added to completely submerge each scaffold piece then incubated overnight and media was subsequently changed every alternate day throughout the experiment.

Determination of viability of cells in collagen scaffolds. Cell-seeded scaffolds were incubated with MTT in a humidified incubator with $5 \% \mathrm{CO}_{2}$ at $37^{\circ} \mathrm{C}$ for $2 \mathrm{~h}$ and the number of cells was qualitatively determined under light microscope. For Hoechst staining, cell-seeded scaffolds were incubated in the dark with $1 \mu \mathrm{g} / \mathrm{ml}$ Hoechst dye for 2 min and stained nuclei were detected by a fluorescence microscope. Quantitative numbers of cells were determined by PicoGreen ${ }^{\circledR}$ assay (Invitrogen) that binds to DNA and measured by fluorescence microplate reader at wavelength of $485 \mathrm{~nm}$ and emission at $520 \mathrm{~nm}$. Metabolic activity was also determined to confirm cell viability by assessing remaining glucose concentration in the conditioned medium. The culture medium $(2 \mu \mathrm{l})$ was assayed daily using Medisafe-mini GR-102 blood glucose meter (Terumo, Japan). The amount of depleted glucose in the medium each day was calculated and reported as cumulative glucose consumption by viable cells.

Histological and morphological evaluation. Cells were fixed in $4 \%$ paraformaldehyde. Specimens were stained with H\&E and mucins for microscopic analysis. For morphological evaluation, cells from both culture systems were fixed with $2.5 \%$ glutaraldehyde ( $\mathrm{pH}$ 7.4). Dried samples were sputter- coated with platinum-palladium and observed under scanning electron microscope (SEM) (Hitachi SEM S-2500, Japan).

2D-PAGE preparation and image analysis. Conditioned medium was collected and cell debris was removed by centrifugation. Supernatant was concentrated by lyophilization and precipitated in $10 \%$ TCA at $4^{\circ} \mathrm{C}$ overnight. Samples were centrifuged at $12,000 \mathrm{rpm}, 4^{\circ} \mathrm{C}$ for $10 \mathrm{~min}$, washed with $25 \% \mathrm{v} / \mathrm{v}$ acetone, and dried by speed vacuum. Specimens were resuspended in lysis buffer containing $9 \mathrm{M}$ urea, 2\% CHAPS, 2\% DTT, 2\% ampholine $\mathrm{pH}$ 3.5-10.0, and 1:500 protease inhibitor (Sigma-Aldrich, USA). Protein concentration was measured by Bradford assay. IPG strips, $7 \mathrm{~cm}$, non-linear, pH 3.0-10.0 gradient (GE Healthcare, USA) were rehydrated with $150 \mathrm{mg}$ protein overnight. IEF was performed at 7,000 $\mathrm{Vh}$, $55 \mathrm{~mA}$ per gel strip using an Ettan IPGphor 3 (GE Healthcare). The IPG strips were equilibrated as previously described (8) and separated in $12.5 \%$ SDS-PAGE followed by CBB R-250 staining. Gels were analyzed by ImageMaster 2D Platinum 7.0 software (GE Healthcare).

In-gel digestion. Protein spots having a volume ratio change of $>1.5$-fold were excised and subjected to in-gel digestion according to the protocol by Srisomsap et al (9). Briefly, gels were destained with $50 \% \mathrm{ACN}$ in $0.1 \mathrm{M} \mathrm{NH}_{4} \mathrm{HCO}_{3}$, reduced with $10 \mathrm{mM}$ DTT, and alkylated with $100 \mathrm{mM}$ iodoacetamide, respectively. Gel pieces were dried then $0.3 \mu \mathrm{g}$ of trypsin (Promega, USA) was added, followed by incubation at $37^{\circ} \mathrm{C}$ overnight and digested peptides were collected for protein identification.

Mass spectrometry. Nanoflow liquid chromatography coupled with the amaZon speed ion trap mass spectrometry (Bruker, USA) was utilized to identify protein spots. A $75 \mu \mathrm{m}$ id $\mathrm{x}$ 100 mm C18 EASY-nLC ${ }^{\text {TM }}$ column (Thermo Scientific, USA) was used. Gradient separation was performed using $0.1 \%$ formic acid in water (solution $\mathrm{A}$ ) and $0.1 \%$ formic acid in ACN (solution B) followed by MS/MS equipped with CaptiveSpray $^{\mathrm{TM}}$ source. Parent mass peaks with a range from 50 to $3,000 \mathrm{~m} / \mathrm{z}$ were selected for MS/MS analysis in which collision energy was fixed at 1,300 V. MS/MS data were processed by Bruker Compass 1.4 software and proteins were then identified using MASCOT with similar search parameters as in a previous study (18). Proteins with molecular weight and $\mathrm{pI}$ consistent to gel spot with MASCOT score $>25$ using p-value $\leq 0.05$ were considered positively identified.

Western blot analysis. Proteins were resolved in $10 \%$ SDS-PAGE and electrophoretically transferred to PVDF membranes (Millipore, USA). The membranes were probed with antibody against human TPI, SFN (1:2,000, Abcam, USA), PGAM1, ENO1, LCP1 (1:1,000, Abcam), $\beta$-tubulin (1:2,000, Cell Signaling Technology, USA), and actin (1:5,000, Sigma, USA) at $4^{\circ} \mathrm{C}$ overnight. Membranes were washed and incubated with corresponding secondary antibody conjugated with HRP (DakoCytomation, Denmark) at room temperature for $1 \mathrm{~h}$. Membranes were probed with ECL (GE Healthcare) and detected by ImageQuant ${ }^{\mathrm{TM}}$ LAS 4000 (GE Healthcare). Then, membranes were stained with CBB R-250 and band intensity was determined to show equal protein loadings. 
A

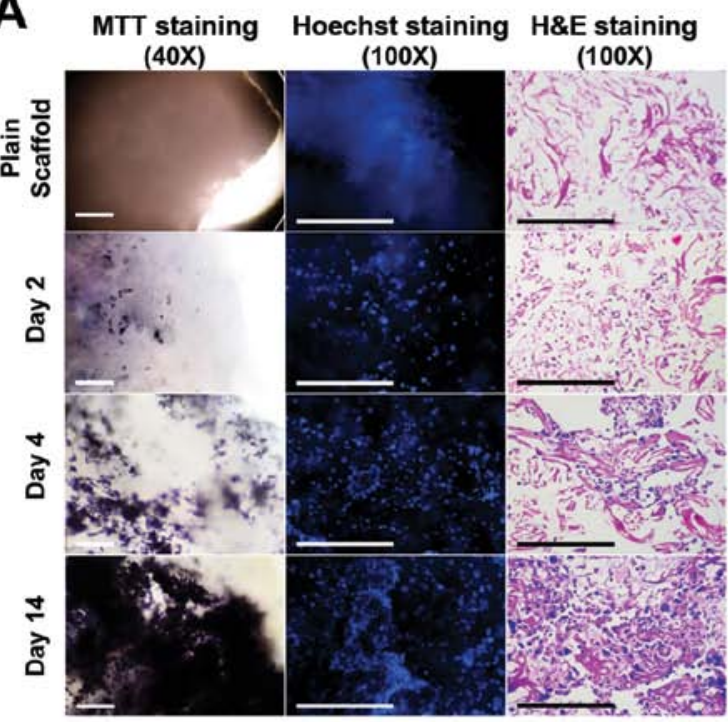

C

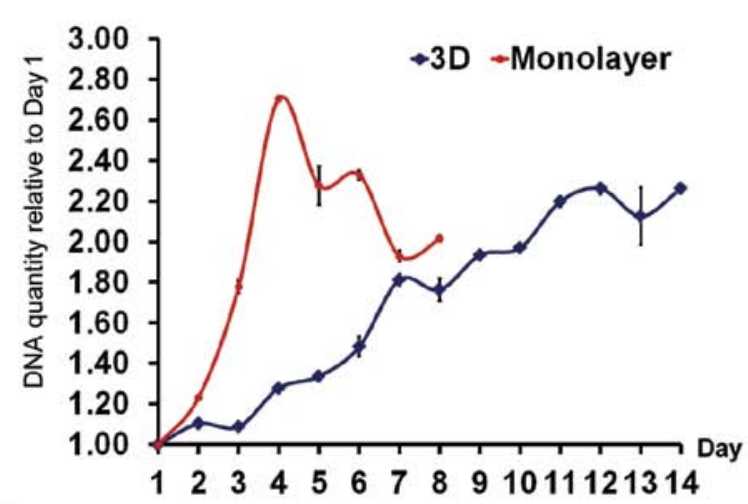

B

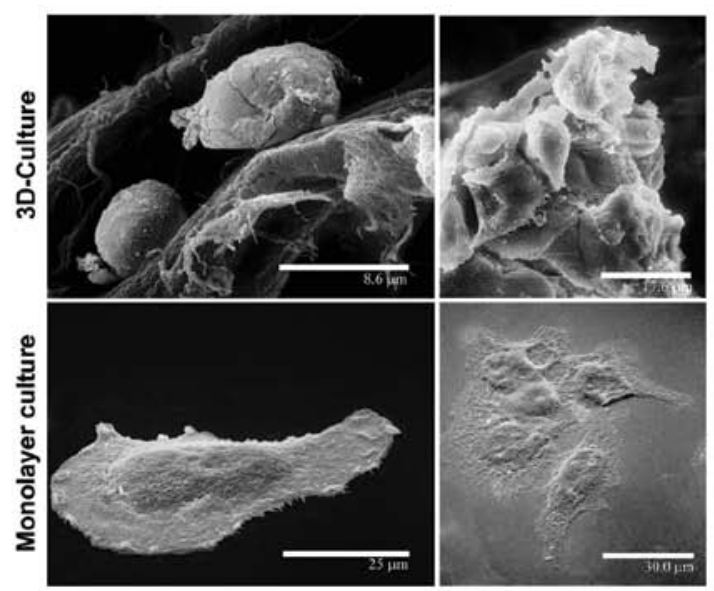

D

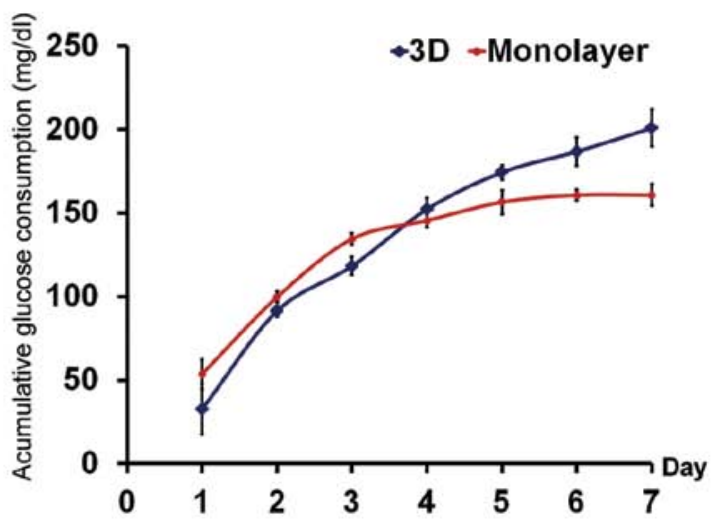

$\mathbf{E}$

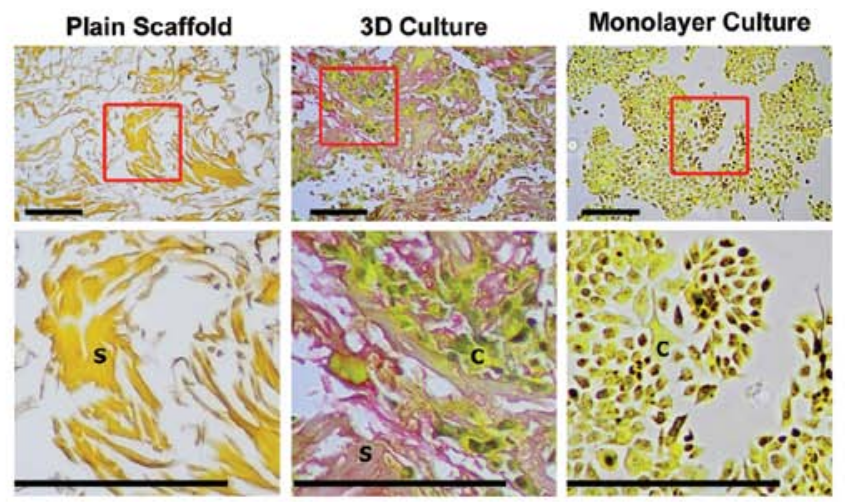

Figure 1. Development and characterization of 3D culture. (A) Cell growth and distribution inside collagen scaffold were determined by MTT (left), Hoechst (middle), and H\&E (right) from day 0 (plain scaffold), days 2, 4 and 14, respectively (scale bars, $100 \mu$ m). (B) Morphological study of HuCCA-1 in monolayer (left) and 3D culture (right) under SEM. (C) Growth pattern of 3D (blue) and monolayer (red) culture at days 1-14 were measured by PicoGreen (data show mean \pm SD from four independent experiments). (D) Comparison of cumulative glucose consumption from 3D (blue) and monolayer (red) culture at days 1-7 (data show mean \pm SD from three independent experiments). (E) Histology of 3D and monolayer culture at day 7 stained with mucicarmine, with plain collagen scaffolds served as negative control.

Statistical analysis. The differences between monolayer and 3D cell culture were analyzed with STATA 10.1 using unpaired t-test. $\mathrm{p}<0.05$ was considered statistically significant.

\section{Results}

Development of HuCCA-1 in scaffold-based $3 D$ culture. The serum-free media cell culture was completely switched to
CDM-HD and morphology observed under a light microscope. There was no significant difference in percentage of viable cells between serum-containing and serum-free cultures. Cell viability was consistently maintained at $>95 \%$.

The serum-free cells were then grown in natural collagenbased scaffold to mimic the dense 3D microenvironment of the in vivo tumor. Growth and localization of cells were studied (Fig. 1A). At the initial phase (day 2), single cells were 
A

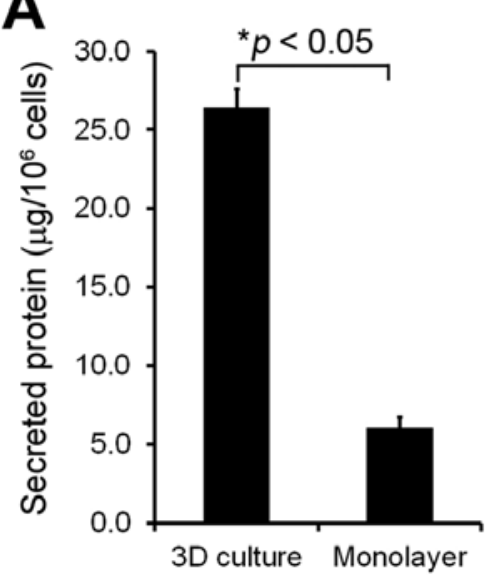

B

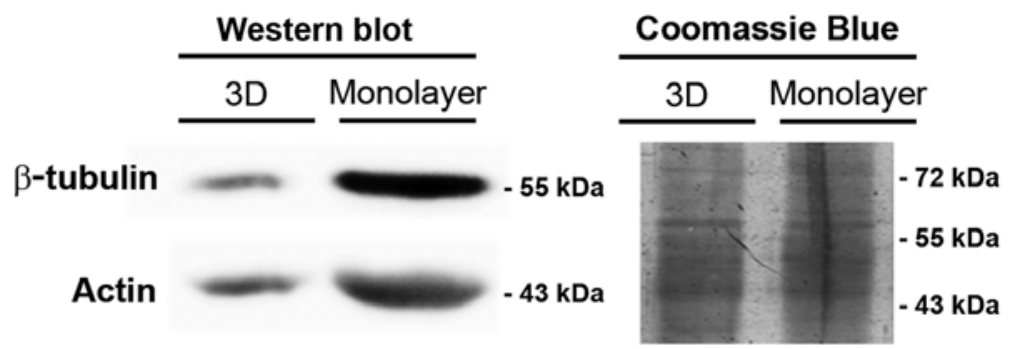

Figure 2. Quantitative and qualitative analysis of secreted protein from day 4. (A) Total secreted protein obtained from 3D and monolayer culture. Data show mean \pm SD from three independent experiments and are reported as $\mu \mathrm{g}$ of protein $/ 10^{6}$ cells. (B) Western blot analysis of $\beta$-tubulin and actin as the quality control of intracellular protein contamination in the conditioned media, using representative membrane stained with CBB R-250 as loading control.

dispersed throughout the scaffold. Then, the cells aggregated into colonies and expanded between the spaces of the spun fibers (days 4 and 14). When cell-seeded scaffolds were stained with MTT (left column), black spots were visible representing cells or small colonies of cells (day 2). Hoechst staining of dsDNA (middle column) showed increasing numbers of blue spots under a fluorescence microscope, indicating increased number of nuclei of cells at increasing time-points (days 2, 4, and 14). H\&E staining (right column) indicated cells with deep blue nuclei and faint pink cytoplasm throughout the pink collagen fibers. These three different methods confirmed progressive growth of HuCCA-1 cells inside collagen scaffolds.

Morphological study under SEM (Fig. 1B) showed alteration of cell shape in 3D culture when compared to monolayer culture. The appearance of cells in the scaffold was round, growing into a dense cluster, whereas the attached cells were flat and expanded as a single thin layer during the 14 days of culture. Cell-cell contacts as well as cell-collagen scaffold contacts were observed in our 3D culture system.

Differential growth patterns between the 3D and monolayer cultures. The growth pattern of cells in different culture systems was determined by PicoGreen assay. Results showed alterations in growth pattern between 3D and monolayer cultures (Fig. 1C). Cells in monolayer culture showed rapid increase in cell proliferation but decreased after reaching the maximum growth in day 4 , while in the 3D culture system, cells showed slower proliferation but maintained a longer growth phase covering a period of over 14 days.

The levels of glucose were monitored and cumulative glucose consumption was calculated compared to starting glucose level in the culture media (Fig. 1D). The pattern of cumulative glucose consumption of cells in 3D culture continuously increased until day 7 , while cumulative glucose consumption of cells in monolayer culture became constant after day 4, reflecting the cessation of growth in monolayer culture after 4 days (Fig. 1C). Thus, cells at day 4 of both culture systems were selected and used for further comparative analyses.

Extracellular matrix deposition in $3 D$ culture. Histological analysis revealed that $3 \mathrm{D}$ culture produced increased level of secreted ECM when compared to monolayer culture. Compared to plain collagen scaffold, a positive result (pink) of mucicarmine staining represented accumulation of mucins in $3 \mathrm{D}$ culture, which was not found in monolayer culture (Fig. 1E).

The quantity and quality of secreted proteins. The protein concentrations extracted from 3D culture were significantly increased $(>5$-fold, $\mathrm{p}<0.05$ ) compared to monolayer culture (Fig. 2A). Thus, the secreted proteins from 3D culture were more extensively enriched and sufficient for further studies.

Contamination of intracellular protein leakage from damaged or dead cells is also a major concern in secretome analysis. To evaluate interference by cell lysis, western blotting of two major cytosolic proteins (actin and $\beta$-tubulin) was performed in conditioned media from both culture systems at day 4. The data indicated significant levels of actin and $\beta$-tubulin in conditioned media obtained from monolayer culture, but decreased presence of these proteins in conditioned media prepared from 3D culture (Fig. 2B). Thus, the lack of these cytoplasmic protein contaminations confirmed the high quality of secreted proteins obtained from 3D culture, making samples from 3D culture ideal for secretome analysis.

Secretome analysis. The secreted proteins from cells grown under the two culture conditions were compared by $2 \mathrm{DE}$ (Fig. 3A). Overall, 431 \pm 14 protein spots were found in 3D culture while $410 \pm 20$ spots were found in monolayer culture. Image analysis showed that 35 secreted protein spots were differentially expressed in 3D culture compared to monolayer culture. Of these, 25 spots were significantly increased in 3D culture and 10 spots were found to be expressed only in 3D culture system (Table I). These proteins were categorized 
A

3D culture

Monolayer
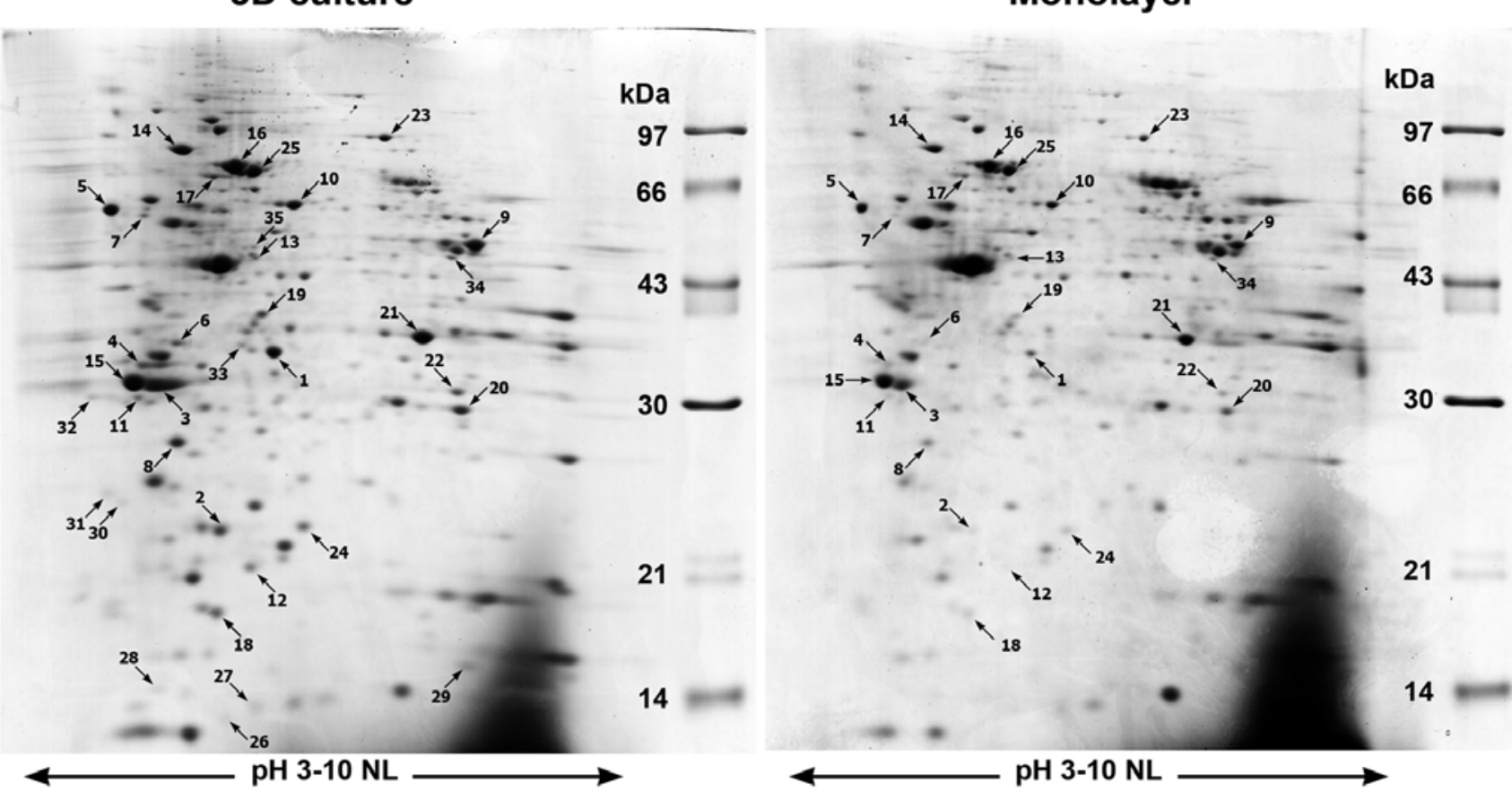

B
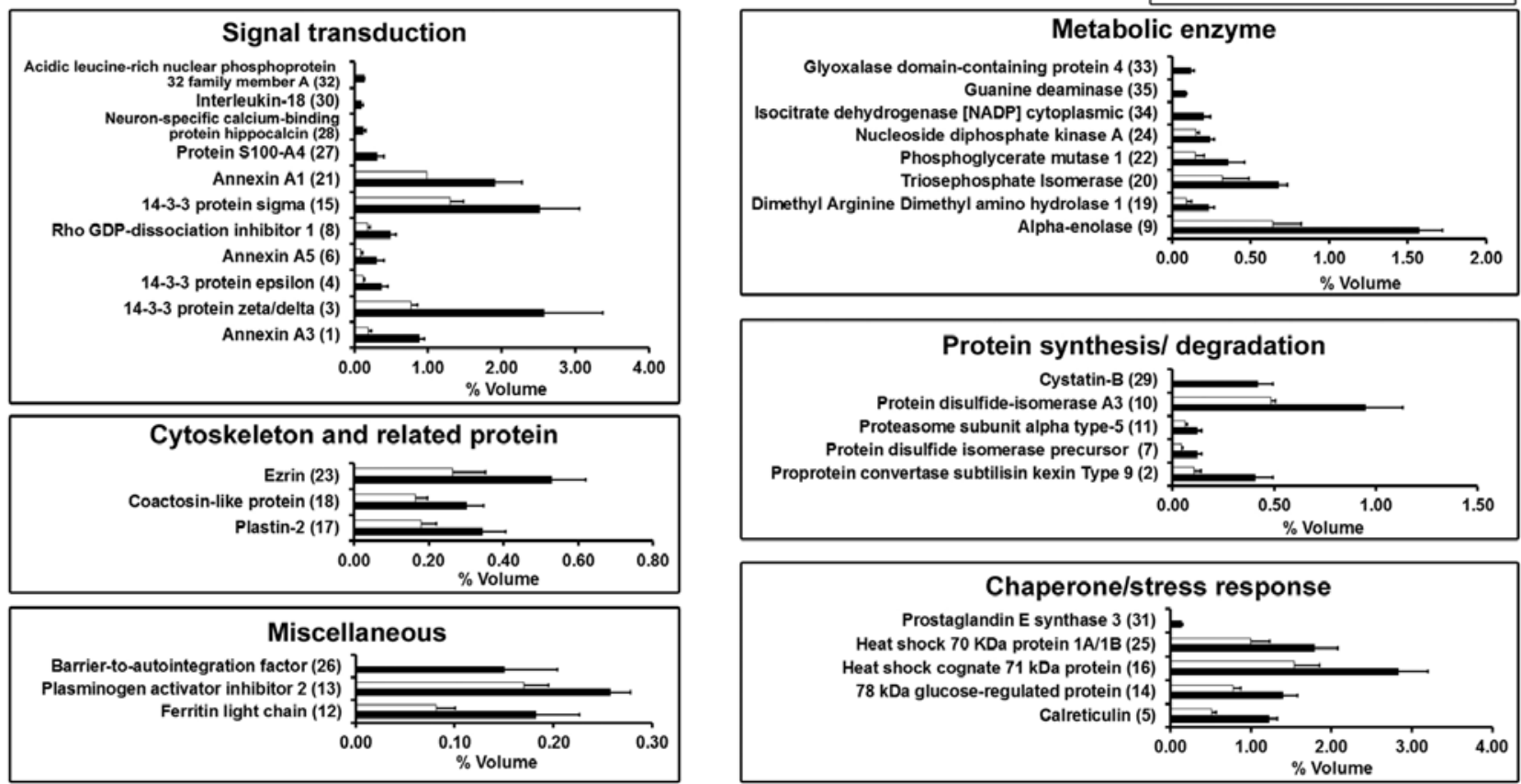

Figure 3. Secretome analysis of HuCCA-1 in 3D and monolayer culture. (A) Representative 2DE patterns of secreted proteins from 3D (left) and monolayer culture (right); arrows indicate protein spots changed by $>1.5$-fold. (B) Significant differentially expressed proteins were categorized into six groups based on biological functions. Black bar shows 3D culture and white bar shows monolayer culture. Spot numbers, as in (A) and Table I, are shown in brackets.

according to their functions, namely: signal transduction, metabolic enzymes, chaperone and stress response, protein synthesis and degradation, cytoskeleton and related, and miscellaneous function proteins (Fig. 3B).

Validation of differential protein expression. Western blot analyses were used to verify the expression of selected secreted proteins which differed considerably between two culture conditions including 14-3-3 $\sigma$ (SFN), triosephosphate isom- erase (TPI), $\alpha$-enolase (ENO1), phosphoglycerate mutase 1 (PGAM1), and L-plastin (LCP1). These results were consistent with the 2DE data in which the expressions of these secreted proteins were higher in 3D than monolayer culture (Fig. 4A). Moreover, the expression of LCP1 in secretomes of CCA cells was used to compare with three liver cancer cell lines (HepG2, SK-HEP-1, and HCC-S102). The result showed that LCP1 was found only in the conditioned media of CCA, but not in the conditioned media of the other liver cell lines (Fig. 4B). 
Table I. Secreted proteins identified in the conditioned media by LC-MS/MS analysis.

\begin{tabular}{|c|c|c|c|c|c|c|c|c|}
\hline $\begin{array}{l}\text { Spot } \\
\text { no. }{ }^{\text {a }}\end{array}$ & $\begin{array}{l}\text { Accession } \\
\text { no. }\end{array}$ & Protein description & Gene name & $\begin{array}{l}\text { Theoritical } \\
(\mathrm{MW} / \mathrm{pI})^{\mathrm{b}}\end{array}$ & Score $^{c}$ & $\begin{array}{l}\text { No. of } \\
\text { peptide }^{\mathrm{d}}\end{array}$ & $\begin{array}{l}\text { Coverage } \\
\quad(\%)\end{array}$ & $\begin{array}{c}\text { Fold } \\
\text { change }^{\mathrm{e}}\end{array}$ \\
\hline 1 & P12429 & Annexin A3 & ANXA3 & $36353 / 5.63$ & 38 & 5 & 13 & $\uparrow 4.71 \pm 0.25$ \\
\hline 2 & Q8NBP7 & $\begin{array}{l}\text { Proprotein convertase subtilisin } \\
\text { type } 9^{f}\end{array}$ & PCSK9 & $14080 / 5.04$ & & & & $\uparrow 3.79 \pm 0.38$ \\
\hline 3 & P63104 & $14-3-3$ protein $\zeta / \delta$ & YWHAZ & $27728 / 4.73$ & 47 & 5 & 20 & $\uparrow 3.35 \pm 0.33$ \\
\hline 4 & P62258 & 14-3-3 protein $\varepsilon$ & YWHAE & $29155 / 4.63$ & 21 & 2 & 5 & $\uparrow 3.19 \pm 0.31$ \\
\hline 5 & P27797 & Calreticulin & CALR & $48112 / 4.29$ & 65 & 3 & 11 & $\uparrow 2.39 \pm 0.13$ \\
\hline 6 & P08758 & Annexin A5 & ANXA5 & $35914 / 4.94$ & 27 & 3 & 7 & $\uparrow 3.36 \pm 0.41$ \\
\hline 7 & P07237 & $\begin{array}{l}\text { Protein disulfide isomerase } \\
\text { precursor }(\mathrm{PDI})^{\mathrm{f}}\end{array}$ & $\mathrm{P} 4 \mathrm{HB}$ & $57100 / 4.78$ & & & & $\uparrow 2.63 \pm 0.23$ \\
\hline 8 & P52565 & Rho GDP-dissociation inhibitor 1 & ARHGDIA & $23193 / 5.02$ & 40 & 3 & 14 & $\uparrow 2.71 \pm 0.27$ \\
\hline 9 & P06733 & $\alpha$-enolase & ENO1 & $47139 / 7.01$ & 70 & 13 & 24 & $\uparrow 2.45 \pm 0.29$ \\
\hline 10 & P30101 & Protein disulfide-isomerase A3 & PDIA3 & $56747 / 5.98$ & 25 & 4 & 7 & $\uparrow 1.95 \pm 0.20$ \\
\hline 11 & P28066 & Proteasome subunit $\alpha$ type- 5 & PSMA5 & $26394 / 4.74$ & 76 & 2 & 7 & $\uparrow 1.94 \pm 0.22$ \\
\hline 12 & P02792 & Ferritin light chain & FTL & $20007 / 5.51$ & 46 & 1 & 4 & $\uparrow 2.24 \pm 0.34$ \\
\hline 13 & P05120 & Plasminogen activator inhibitor 2 & SERPINB2 & $46566 / 5.46$ & 92 & 5 & 12 & $\uparrow 1.51 \pm 0.17$ \\
\hline 14 & P11021 & 78-kDa glucose-regulated protein & HSPA5 & $72288 / 5.07$ & 301 & 13 & 20 & $\uparrow 1.79 \pm 0.17$ \\
\hline 15 & P31947 & $14-3-3$ protein $\sigma$ & SFN & $27757 / 4.68$ & 172 & 5 & 16 & $\uparrow 1.93 \pm 0.26$ \\
\hline 16 & P11142 & Heat shock cognate $71-k D a$ protein & HSPA8 & $70854 / 5.37$ & 382 & 17 & 22 & $\uparrow 1.84 \pm 0.24$ \\
\hline 17 & P13796 & Plastin-2 & LCP1 & $70244 / 5.29$ & 363 & 10 & 18 & $\uparrow 1.91 \pm 0.28$ \\
\hline 18 & Q14019 & Coactosin-like protein & COTL1 & $15935 / 5.54$ & 107 & 7 & 33 & $\uparrow 1.82 \pm 0.25$ \\
\hline 19 & O94760 & $\begin{array}{l}\text { Dimethyl arginine dimethyl amino- } \\
\text { hydrolase } 1\end{array}$ & DDAH1 & $31102 / 5.53$ & 240 & 7 & 26 & $\uparrow 2.54 \pm 0.36$ \\
\hline 20 & P60174 & Triosephosphate isomerase & TPI1 & $30772 / 5.65$ & 386 & 9 & 37 & $\uparrow 2.12 \pm 0.53$ \\
\hline 21 & P04083 & Annexin A1 & ANXA1 & $38690 / 6.57$ & 253 & 12 & 29 & $\uparrow 1.94 \pm 0.31$ \\
\hline 22 & P18669 & Phosphoglycerate mutase 1 & PGAM1 & $28786 / 6.67$ & 76 & 3 & 10 & $\uparrow 2.43 \pm 0.51$ \\
\hline \multirow[t]{2}{*}{23} & P15311 & Ezrin & EZR & $69370 / 5.94$ & 335 & 21 & 26 & $\uparrow 2.00 \pm 0.37$ \\
\hline & P26038 & Moesin & MSN & $67778 / 6.08$ & 128 & 9 & 10 & \\
\hline 24 & P15531 & Nucleoside diphosphate kinase A & NME1 & $17138 / 5.38$ & 95 & 5 & 30 & $\uparrow 1.58 \pm 0.16$ \\
\hline 25 & P08107 & Heat shock 70-kDa protein 1A/1B & HSPA1A & $70009 / 5.48$ & 556 & 15 & 24 & $\uparrow 1.79 \pm 0.29$ \\
\hline 26 & O75531 & Barrier-to-autointegration factor & BANF1 & $10052 / 5.81$ & 125 & 2 & 21 & $\mathrm{~N} / \mathrm{A}$ \\
\hline 27 & P26447 & Protein S100-A4 & S100A4 & $11721 / 5.85$ & 57 & 1 & 7 & N/A \\
\hline 28 & P84074 & $\begin{array}{l}\text { Neuron-specific calcium-binding } \\
\text { protein hippocalcin }\end{array}$ & $\mathrm{HPCA}$ & $22413 / 4.87$ & 27 & 1 & 5 & N/A \\
\hline 29 & P04080 & Cystatin-B & CSTB & $11133 / 6.96$ & 29 & 2 & 12 & N/A \\
\hline 30 & Q14116 & Interleukin-18 & IL18 & $22312 / 4.54$ & 78 & 3 & 12 & N/A \\
\hline 31 & Q15185 & Prostaglandin E synthase 3 & PTGES3 & $18685 / 4.35$ & 82 & 2 & 12 & N/A \\
\hline 32 & P39687 & $\begin{array}{l}\text { Acidic leucine-rich nuclear } \\
\text { phospho-protein } 32 \text { family } \\
\text { member A }\end{array}$ & ANP32A & $28568 / 3.99$ & 92 & 5 & 16 & N/A \\
\hline 33 & Q9HC38 & $\begin{array}{l}\text { Glyoxalase domain-containing } \\
\text { protein } 4\end{array}$ & GLOD4 & $34771 / 5.40$ & 124 & 5 & 17 & N/A \\
\hline 34 & O75874 & $\begin{array}{l}\text { Isocitrate dehydrogenase (NADP) } \\
\text { cytoplasmic }\end{array}$ & IDH1 & $46630 / 6.53$ & 151 & 7 & 16 & N/A \\
\hline 35 & Q9Y2T3 & Guanine deaminase & GDA & $50971 / 5.44$ & 104 & 6 & 11 & N/A \\
\hline
\end{tabular}

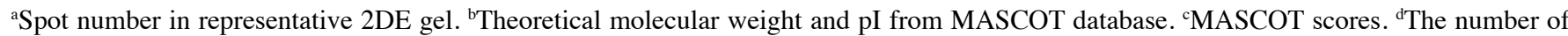
unique peptides identified by MS/MS. ${ }^{~}$ Volume ratio of protein spot intensity in 3D relative to monolayer culture calculated by ImageMaster

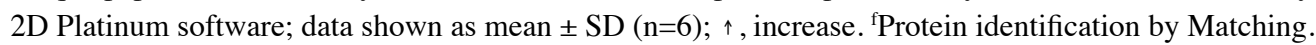


A

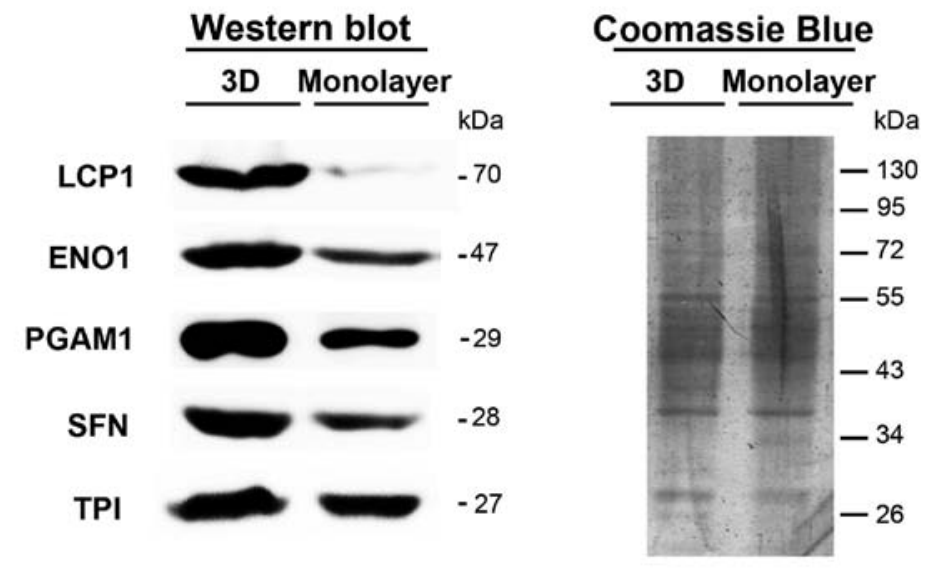

B

Liver cancer

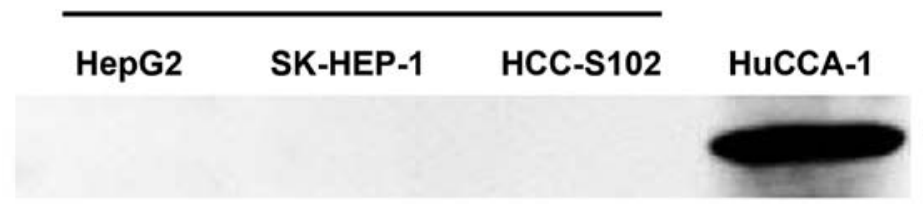

$70 \mathrm{kDa}$

Figure 4. Validation of the selected enrichment of CCA secretomes. (A) Western blot analysis of LCP1, ENO1, PGAM1, SFN and TPI obtained from secreted protein of 3D and monolayer culture. Membrane stained with CBB R-250 represents loading control. (B) Western blot analysis of LCP1 obtained from conditioned media samples of CCA (HuCCA-1) compared with three liver cancer cell lines (HepG2, SK-HEP-1 and HCC-S102).

\section{Discussion}

We suggest that the developed 3D culture model of CCA is suitable for secretome analysis and may be a useful method for discovery of novel biomarkers. Here, scaffold-based 3D culture of HuCCA-1 cells in serum-free condition was analyzed. The result suggests the advantage of $3 \mathrm{D}$ culture model over monolayer culture in providing long-term culture. Since CCA is an extremely heterogeneous cancer (19), long-term culture might facilitate differentiation and proliferation of different cell types providing a more realistic tumor population. SEM technique also revealed that the morphology of HuCCA-1 cells inside the scaffold were ellipsoid in shape similar to simple cuboidal epithelium, the morphology of cholangiocytes in vivo. Moreover, cells in 3D culture mimic a more natural cell-cell and cell-ECM behavior. Interestingly, Chiarini et al reported the induction of ductal-like structure of cholangiocytes in vitro using the collagen gel scaffold (20). Taken together, this evidence indicates the induction of in vivo-like cell morphology, which shows promising advantage over the flat cell morphology found in monolayer culture. The cell-ECM interaction in $3 \mathrm{D}$ culture also allows the collagen fiber to absorb extracellular matrix mucin secreted by HuCCA-1 cells. The capability of cancer cells grown in 3D culture to enhance ECM deposition was reported by Pruksakorn et al using this scaffold for 3D culture of HepG2 cells and they found increased levels of sulfated-glycosaminoglycans (17). Mucin expression appears to be associated with intrahepatic bile duct development and relate to progression of CCA (21). Thus, culture of HuCCA-1 cells in a 3D scaffold makes it feasible to accumulate crucial cell-ECM interaction, which may confer a better in vivo-like environment than conventional cell culture.
Our findings provide that scaffold-based 3D cell culture yields superior quantity and quality of secreted proteins. Our group previously reported the analysis of secreted protein from HuCCA-1 cell culture in hollow fiber bioreactor (16). Consistent with this study, secreted proteins from hollow fiber system, which also aims to mimic in vivo microenvironment, were enhanced compared to monolayer culture. In agreement with this notion, rabbit mammary cells will synthesize, store, and secrete fat and milk proteins in 3D culture but cannot do so in monolayer culture (22). In this case, researchers propose that cell polarity and differentiation, which are important for milk secretion, were influenced by cell-matrix interaction in 3D culture. Since cholangiocytes are polarized cell-like mammary cells, it is possible that cell shape and cell-collagen interactions might affect cell polarity and secretion ability of the cells. Thus, further supporting our study that scaffoldbased 3D cell cultures are better suited for secretome analysis and the differentially expressed proteins would be a more accurate reflection of proteins secreted in vivo.

In this investigation, upregulation of 14-3-3 $\sigma$ or stratifin (SFN) in 3D culture compared to monolayer culture was confirmed by Western blot analysis. This signaling protein is an intracellular, phosphoserine binding protein that is thought to be involved in cancer development of several organs. We recently reported the upregulation of this protein and its important role in anoikis resistance of CCA cells (18). Interestingly, overexpression of SFN appears to be correlated with increased tumor progression and poor prognosis in colorectal (23) and gastric cancer (24). Since SFN was intensely secreted in 3D culture of HuCCA-1, this protein might also play an important role in the tumor microenvironment of the cells and may be a potential biomarker in CCA. Additionally, upregulation of 
selected glycolytic proteins such as TPI, PGAM1, and ENO1 in $3 \mathrm{D}$ culture were confirmed. We hypothesize that this is due to the enhanced Warburg phenomenon as reported in other studies such as osteosarcoma (25) and hepatocellular carcinoma (17).

Multiple proteins associated with aggressive cancer phenotypes were found to be upregulated. TPI is a glycolytic enzyme that catalyzes the reversible interconversion of dihydroxyacetone phosphate (DHAP) and D-glyceraldehyde 3-phosphate (G3P). Significant upregulation of TPI has been found in various cancers including breast cancer (26) and brain metastatic tissues of endometrial and ovarian cancers (27). Another glycolytic enzyme considerably upregulated in 3D culture is PGAM1 which reversibly catalyzes the conversion of 3-phosphoglycerate (3PG) into 2-phosphoglycerate (2PG). Marked upregulation of PGAM1 is strongly correlated with poor differentiation of hepatocellular carcinoma patients (28). We also found high expression of ENO1. This enzyme catalyzes 2-phosphoglycerate (2-PG) to phosphoenolpyruvate (PEP) in the glycolysis pathway. Upregulated ENO1 in CCA tissue is significantly associated with poor prognosis and tumor invasion (29).

Promising biomarkers need to be significantly expressed and ideally specific or capable of differentiating between types of cancer. In our study, one such protein was identified to be LCP1. This protein is generally expressed in rapidly movable cells such as leukocytes and cancer cells may gain the ability to metastasize to other parts of the body by expressing LCP1 (30). This protein is specifically expressed in non-hematopoietic cancers including ovarian (31) and colorectal cancer (32). In addition, Lin et al reported that $68 \%$ of cancers derived from epithelia express LCP1 (33). Accordingly, LCP1 was a main focus among validated secreted proteins because of its functional involvement in cancer metastasis and lack of research on the role of LCP1 in CCA. Though LCP1 is expressed in several types of cancers, the level of LCP1 mRNA expression in HepG2 could not be detected by northern blot analysis (33) and RT-PCR (34) while SK-HEP-1 showed only trace levels of LCP1 mRNA detectable by RT-PCR (34). Thus, LCP1 has the potential to be used as biomarker for the discrimination of CCA from $\mathrm{HCC}$ and needs to be evaluated further in clinical specimens such as serum and tissues from CCA patients.

Additionally, our data revealed that some proteins can be detected only in the secreted fraction from 3D culture, suggesting that they may require the 3D microenvironment for expression and/or secretion. Interleukin-18 has been shown to be associated with various cancers including renal cancer (35), and hepatocellular carcinoma (36). By using 3D culture based secretome analysis, this is the first evidence of IL-18 secretion in the HuCCA-1 cell line. Therefore, this protein might be another promising candidate as a biomarker of CCA and should be studied further.

In conclusion, we are the first group to report the successful development of serum-free intrahepatic CCA cell line cultured in a collagen scaffold-based 3D system. Our study shows that characteristics of HuCCA-1 cells were modified in response to the $3 \mathrm{D}$ environment allowing cells to behave in a more in vivo-like manner where cell contact (cell-cell or cell-ECM) is drastically different from conventional cell culture. Consequently, the cells in this environment should behave and secrete proteins that mimic in vivo CCA more accurately allowing for discovery of promising biomarkers for CCA.

\section{Acknowledgements}

We would like to thank Dr Titipatima Sakulterdkiat for constructive feedback in writing this manuscript. This study was supported by the Chulabhorn Research Institute, Chulabhorn Graduate Institute, and the Center of Excellence on Environmental Health, Toxicology and Management of Chemicals, Bangkok, Thailand.

\section{References}

1. Khan SA, Thomas HC, Davidson BR and Taylor-Robinson SD: Cholangiocarcinoma. Lancet 366: 1303-1314, 2005.

2. Khan SA, Toledano MB and Taylor-Robinson SD: Epidemiology, risk factors, and pathogenesis of cholangiocarcinoma. HPB 10: 77-82, 2008.

3. Sripa B, Kaewkes S, Sithithaworn P, et al: Liver fluke induces cholangiocarcinoma. PLoS Med 4: e201, 2007.

4. Leelawat K, Narong S, Wannaprasert J and Ratanashu-ek T: Prospective study of MMP7 serum levels in the diagnosis of cholangiocarcinoma. World J Gastroenterol 16: 4697-4703, 2010.

5. Mott JL and Gores GJ: Targeting IL-6 in cholangiocarcinoma therapy. Am J Gastroenterol 102: 2171-2172, 2007.

6. Shigehara K, Yokomuro S, Ishibashi O, et al: Real-time PCR-based analysis of the human bile microRNAome identifies miR-9 as a potential diagnostic biomarker for biliary tract cancer. PLoS One 6: e23584, 2011.

7. Makridakis M and Vlahou A: Secretome proteomics for discovery of cancer biomarkers. J Proteomics 73: 2291-2305, 2010.

8. Srisomsap C, Sawangareetrakul P, Subhasitanont P, et al: Proteomic analysis of cholangiocarcinoma cell line. Proteomics 4: 1135-1144, 2004.

9. Srisomsap C, Sawangareetrakul P, Subhasitanont P, et al: Proteomic studies of cholangiocarcinoma and hepatocellular carcinoma cell secretomes. J Biomed Biotechnol 2010: 437143, 2010.

10. Hutmacher DW, Loessner D, Rizzi S, Kaplan DL, Mooney DJ and Clements JA: Can tissue engineering concepts advance tumor biology research? Trends Biotechnol 28: 125-133, 2010.

11. Pampaloni F, Reynaud EG and Stelzer EH: The third dimension bridges the gap between cell culture and live tissue. Nat Rev Mol Cell Biol 8: 839-845, 2007.

12. Yamada KM and Cukierman E: Modeling tissue morphogenesis and cancer in 3D. Cell 130: 601-610, 2007.

13. Weigelt B and Bissell MJ: The need for complex 3D culture models to unravel novel pathways and identify accurate biomarkers in breast cancer. Adv Drug Deliv Rev 69-70: 42-51. 2014.

14. Sirisinha S, Tengchaisri T, Boonpucknavig S, Prempracha N, Ratanarapee S and Pausawasdi A: Establishment and characterization of a cholangiocarcinoma cell line from a Thai patient with intrahepatic bile duct cancer. Asian Pac J Allergy Immunol 9: 153-157, 1991.

15. Laohathai $\mathrm{K}$ and Bhamarapravati $\mathrm{N}$ : Culturing of human hepatocellular carcinoma. A simple and reproducible method. Am J Pathol 118: 203-208, 1985.

16. Weeraphan C, Diskul-Na-Ayudthaya P, Chiablaem K, et al: Effective enrichment of cholangiocarcinoma secretomes using the hollow fiber bioreactor culture system. Talanta 99: 294-301, 2012.

17. Pruksakorn D, Lirdprapamongkol K, Chokchaichamnankit D, et al: Metabolic alteration of HepG2 in scaffold-based 3-D culture: proteomic approach. Proteomics 10: 3896-3904, 2010.

18. Khongmanee A, Lirdprapamongkol K, Tit-oon P, Chokchaichamnankit D, Svasti J and Srisomsap C: Proteomic analysis reveals important role of 14-3-3sigma in anoikis resistance of cholangiocarcinoma cells. Proteomics 13: 3157-3166, 2013. 
19. Cardinale V, Carpino G, Reid L, Gaudio E and Alvaro D: Multiple cells of origin in cholangiocarcinoma underlie biological, epidemiological and clinical heterogeneity. World J Gastrointest Oncol 4: 94-102, 2012

20. Chiarini LB, Takiya CM, Borojevic R and Monteiro AN: Long-term culture of cholangiocytes from liver fibro-granulomatous lesions. BMC Gastroenterol 6: 13, 2006.

21. Mall AS, Tyler MG, Ho SB, et al: The expression of MUC mucin in cholangiocarcinoma. Pathol Res Pract 206: 805-809, 2010.

22. Haeuptle MT, Suard YL, Bogenmann E, Reggio H, Racine L and Kraehenbuhl JP: Effect of cell shape change on the function and differentiation of rabbit mammary cells in culture. J Cell Biol 96 1425-1434, 1983.

23. Perathoner A, Pirkebner D, Brandacher G, et al: 14-3-3sigma expression is an independent prognostic parameter for poor survival in colorectal carcinoma patients. Clin Cancer Res 11: 3274-3279, 2005.

24. Zhou WH, Tang F, Xu J, et al: Aberrant upregulation of 14-3-3o expression serves as an inferior prognostic biomarker for gastric cancer. BMC Cancer 11: 397, 2011.

25. Santini MT, Rainaldi G, Romano R, et al: MG-63 human osteosarcoma cells grown in monolayer and as three-dimensional tumor spheroids present a different metabolic profile: a (1)H NMR study. FEBS Lett 557: 148-154, 2004.

26. Thongwatchara P, Promwikorn W, Srisomsap C, Chokchaichamnankit D, Boonyaphiphat $\mathrm{P}$ and Thongsuksai P: Differential protein expression in primary breast cancer and matched axillary node metastasis. Oncol Rep 26: 185-191, 2011.

27. Yoshida A, Okamoto N, Tozawa-Ono A, et al: Proteomic analysis of differential protein expression by brain metastases of gynecological malignancies. Hum Cell 26: 56-66, 2013.
28. Ren F, Wu H, Lei Y, et al: Quantitative proteomics identification of phosphoglycerate mutase 1 as a novel therapeutic target in hepatocellular carcinoma. Mol Cancer 9: 81, 2010.

29. Yonglitthipagon P, Pairojkul C, Bhudhisawasdi V, Mulvenna J, Loukas A and Sripa B: Proteomics-based identification of alphaenolase as a potential prognostic marker in cholangiocarcinoma. Clin Biochem 45: 827-834, 2012.

30. Shinomiya $\mathrm{H}$ : Plastin family of actin-bundling proteins: its functions in leukocytes, neurons, intestines, and cancer. Int J Cell Biol 2012: 213492, 2012.

31. Kang S, Shim HS, Lee JS, et al: Molecular proteomics imaging of tumor interfaces by mass spectrometry. J Proteome Res 9: 1157-1164, 2010.

32. Ang CS and Nice EC: Targeted in-gel MRM: a hypothesis driven approach for colorectal cancer biomarker discovery in human feces. J Proteome Res 9: 4346-4355, 2010.

33. Lin CS, Park T, Chen ZP and Leavitt J: Human plastin genes. Comparative gene structure, chromosome location, and differential expression in normal and neoplastic cells. J Biol Chem 268: 2781-2792, 1993.

34. Park T, Chen ZP and Leavitt J: Activation of the leukocyte plastin gene occurs in most human cancer cells. Cancer Res 54: 1775-1781, 1994

35. Saenz-Lopez P, Carretero R, Vazquez F, et al: Impact of interleukin-18 polymorphisms-607 and -137 on clinical characteristics of renal cell carcinoma patients. Hum Immunol 71: 309-313, 2010.

36. Tangkijvanich $\mathrm{P}$, Thong-Ngam $\mathrm{D}$, Mahachai $\mathrm{V}$, Theamboonlers $\mathrm{A}$ and Poovorawan Y: Role of serum interleukin-18 as a prognostic factor in patients with hepatocellular carcinoma. World J Gastroenterol 13: 4345-4349, 2007. 